\title{
External Laryngocoele or Anterior Jugular Vein Aneurysm (A Rare Clinical Entity): A Paradox Solved by A Few Simple Non-Invasive Radiological Tests
}

Somesh Mozumder, ${ }^{1}$ Shirish Dubey, ${ }^{1}$ Aniruddha Dam, ${ }^{1}$ Anup Kumar Bhowmick ${ }^{1}$

\begin{abstract}
Introduction
The differential diagnosis of a cystic upper neck mass that becomes prominent on coughing, straining, breath holding, or Valsalva manoeuvre includes mediastinal tumours and cysts, external laryngeal diverticula, and jugular venous aneurysms. Jugular venous aneurysms, while extremely rare, must be considered. We report the fifth case of anterior jugular aneurysm in an adult patient.

Case Report

A 55 year old female patient presented with a swelling in the upper part of right side of neck near the greater cornu of hyoid bone. The swelling increased with Valsalva, straining and while stooping forward. Clinically it was thought to be an external laryngocoele. However Colour Doppler Ultra-sonography and C.T angiogram of neck established it as of anterior jugular venous aneurysm by distinguishing from external laryngocoele.

Discussion

Patient presenting with unilateral cystic swelling in upper neck at upper border of thyroid cartilage which clinically bears the common provisional diagnosis of external laryngocele must be differentiated radiologically from anterior jugular venous aneurysm, (though rare in occurrence) to avoid a catastrophic incident during surgery.

Keywords

Aneurysm; Neck; Jugular Vein.
\end{abstract}

ABSTRACT

$\mathrm{T}$ The differential diagnosis of a cystic upper neck mass that becomes prominent on coughing, straining, breath holding, or Valsalva manoeuvre includes mediastinal tumours and cysts, external laryngeal diverticula and laryngocele, and jugular venous aneurysms. ${ }^{1,2,3}$ Primary isolated venous aneurysm involving the low pressure superior vena caval reservoir system is extremely rare, particularly in the absence of an underlying cardiovascular disease or trauma.

Anterior jugular phlebectasia or aneurysm is very rare, with only seven cases published in medical literature, out of which three were in a paediatric population. ${ }^{4} \mathrm{We}$ report the fifth case of anterior jugular aneurysm in an adult patient.

Recognition that jugular venous aneurysms are a differential diagnosis for neck masses, and that Anterior
Jugular Venous aneurysm may occur in isolation is important to avoid diagnostic confusion.

The diagnosis is suggested by clinical features and can be confirmed by non-invasive radiology. Surgery is indicated to avoid thromboembolic manifestations and for cosmetic reasons. ${ }^{5}$

\section{Case report}

A 55 year old female patient from rural Bengal presented

1 - Department of ENT, Chittaranjan National Cancer Institute, Kolkata

Corresponding author:

Dr Somesh Mozumder

email: drsomeshmozumder@gmail.com 


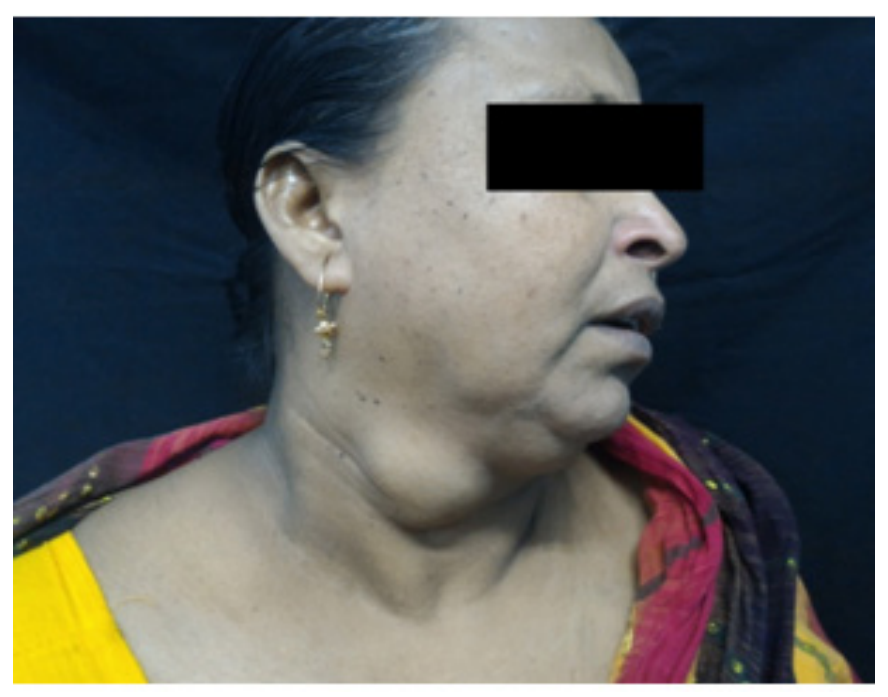

Fig.1. Clinical photograph

with a swelling in the upper part of right side of neck near the greater cornu of hyoid bone. The swelling was incidental in onset, gradually increasing in size, soft, cystic, compressible, non translucent, non fluctuant, non expansile, but grows in size with Valsalva manoeuvre, straining and while stooping forward. Patient gave a history of regular conch blowing before the onset of the swelling. Thus, clinically it was thought to be an

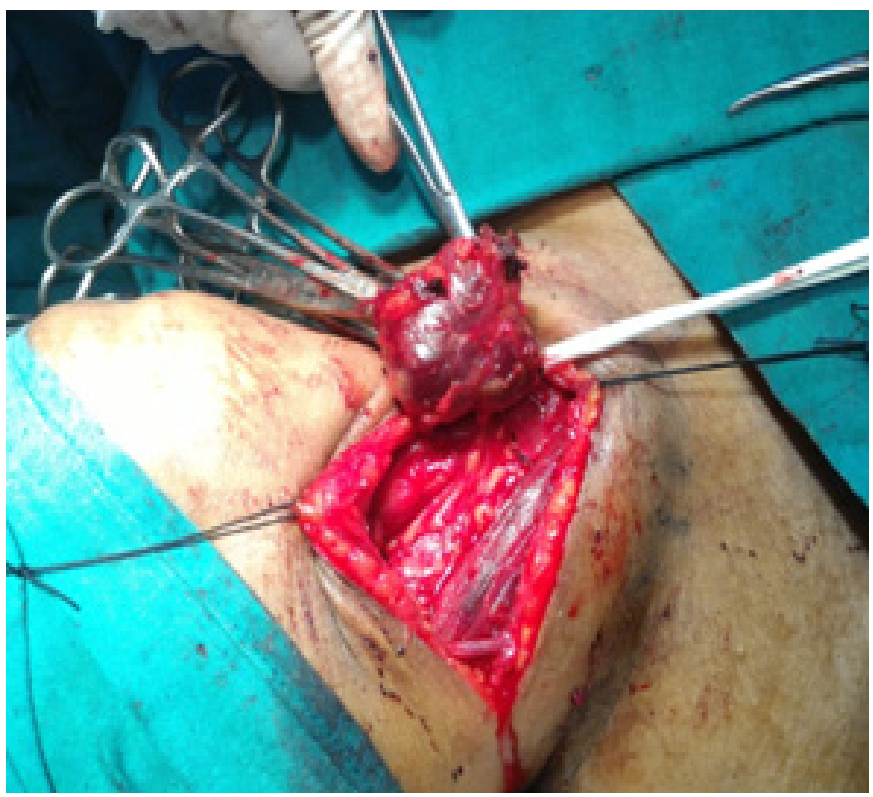

Fig.3. Aneurysm is being removed.

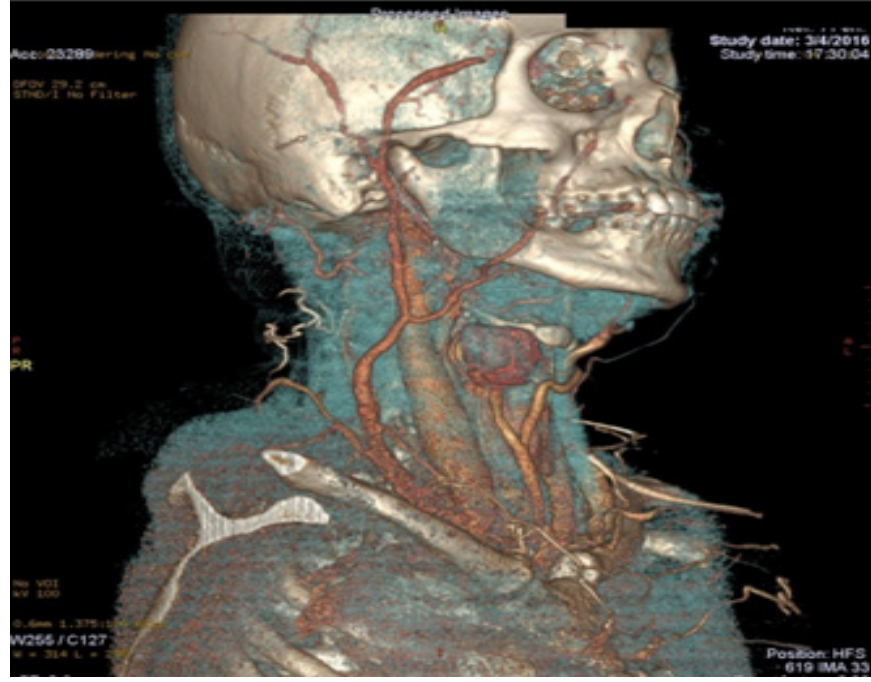

Fig.2. CT angiogram of neck showed aneurysmal swelling of right anterior jugular vein.

external laryngocoele, but x-ray soft tissue neck (AP and lateral view) with and without Valsalva revealed a soft tissue swelling which is non translucent.

Colour Doppler Ultra-sonography of neck revealed the swelling was getting blood filled during Valsalva manoeuvre. CT Angiogram was carried out which showed aneurysmal swelling of right Anterior Jugular Vein without any other feeder.

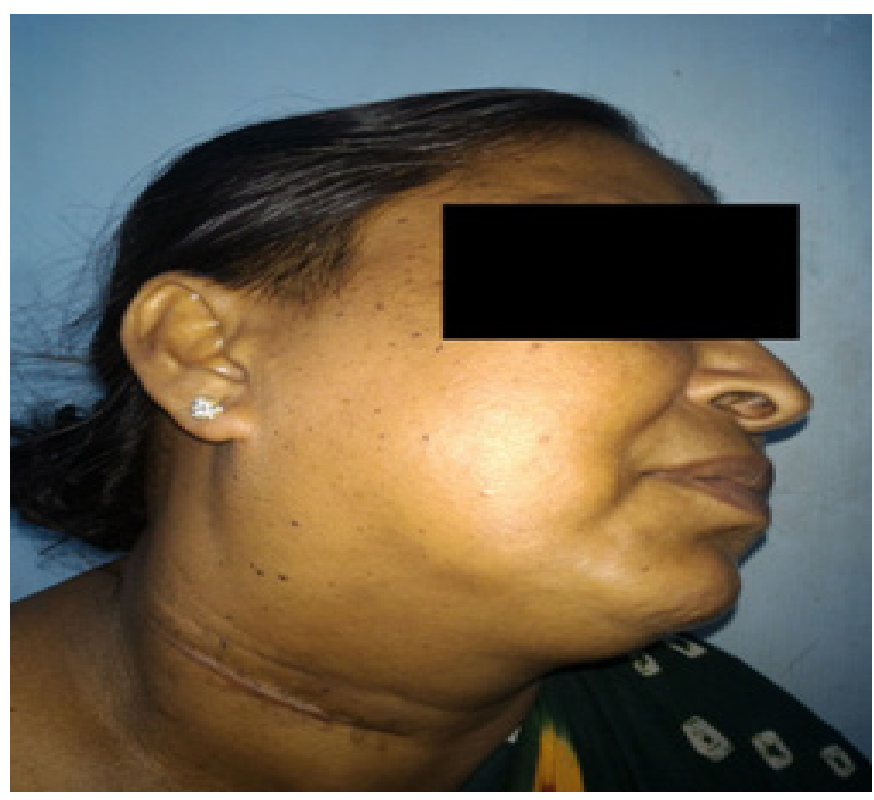

Fig.4: Post Operative photograph 
Under general anaesthesia the aneurysm was removed after clamping the anterior jugular vein.

The patient was kept under regular follow up for 6 months with no signs of recurrence.

\section{Discussion}

Venous dilatation in the neck involves the internal, external and anterior jugular vein, in descending order of frequency. ${ }^{6}$ Clinically, by a careful process of elimination, the preoperative diagnosis can often be accurately established. Aneurysm of the anterior jugular vein mostly presents as a painless swelling. Thrombosis within the aneurysm can produce pain in the swelling and symptoms secondary to pressure effect on surrounding structures. ${ }^{2}$

The radiological investigations may be in the form of digital soft tissue $\mathrm{x}$-ray neck (AP and lateral view) with and without Valsalva, Doppler ultra-sonogram to sophisticated tools such as CT angiography, and magnetic resonance angiography.

Digital soft tissue $\mathrm{x}$-ray neck (AP and lateral view) helps to differentiate between a cystic air filled sac and solid soft tissue lesion in upper neck and thus virtually eliminates the differential diagnosis of external laryngocoele.

Ultrasound with Doppler allows differentiation between cystic and solid lesions, differentiation of vascular from non-vascular lesions, identification of site of origin of the lesion, and its relationship with the surrounding structures in the neck. ${ }^{7,8}$

CT-angiogram is the final investigation used to delineate the type, origin of lesion and feeding vessels therefore helping to prepare the surgical plan5.

Asymptomatic aneurysms can be managed expectantly with reassurance and regular follow-up. Surgical excision is offered for either cosmetic reasons or a painful aneurysm secondary to thrombosis or phlebitis of the jugular venous system. ${ }^{9}$ Surgical resection also eliminates the theoretical risk of aneurysmal rupture, pulmonary embolism and allows for histopathological diagnosis. ${ }^{10}$ A symptomatic jugular venous aneurysm can be safely managed by excision and ligation. ${ }^{5}$

\section{Conclusion}

Patient presenting with unilateral cystic swelling in upper neck at upper border of thyroid cartilage which is non-tender, soft, and non-pulsatile and enlarges with straining, crying, sneezing, or Valsalva manoeuvre bears the provisional diagnosis of external laryngocele, but must be differentiated radiologically from anterior jugular venous aneurysm (though rare in occurrence) to avoid a catastrophic incident during surgery.

\section{References}

1. Zohar Y, Ben-Tovim R, Talmi YP. Phlebectasia of the jugular system. J Craniomaxillofac Surg. 1989; 17: 96-8

2. LaMonte SJ, Walker EA, Moran WB. Internal jugular phlebectasia. A clinicoroentgenographic diagnosis Arch Otolaryngol 1976; 102: 706-8

3. Nwako FA, Agugua NE, Udeh CA, Osuorji RI. Jugular phlebectasia. J Pediatr Surg. 1989; 24: 303-5

4. Gupta P, Bhargava S, Bhargava SK, Gupta R. Anterior Jugular Phlebectasia: Diagnosis by Multislice CT JIMSA 2013; 26 (1): 57

5. Calligaro KD, Ahmad S, Dandora R, Dougherty MJ, Savarese $\mathrm{RP}$, Doerr KJ et al. Venous aneurysms: surgical indications and review of the literature. Surgery 1995; 117: 1-6

6. LaMonte SJ, Walker EA, Moran WB. Internal jugular phlebectasis. A clinicoroentgenographic diagnosis. Arch Otolaryngol. 1976; 102: 706-8

7. Lee HY, Yoo SM, Song IS, Yu H, Lee JB. Sonographic diagnosis of a saccular aneurysm of the internal jugular vein. $\mathrm{J}$ Clin Ultrasound. 2007; 35: 94-6

8. Wen C, Liu R, Liu M, Sun L, Wang Z, Tang J. Saccular neck venous aneurysm on color duplex sonography: an analysis of 12 cases. Clin Imaging 2009; 33:424-9

9. Ascher E, Salles-Cunha S, Hingorani A. Morbidity and mortality associated with internal jugular vein thromboses. Vasc Endovascular Surg. 2005; 39:335-9

10. Ioannou CV, Kostas T, Tsetis D, Georgakarakos E, Gionis M, Katsamouris AN. External jugular vein aneurysm: A source of thrombotic complications Int Angiol. 2010; 29: 284-5. 